\title{
Readily Dispersible Chemically Functionalized Reduced Graphene Oxide Nanosheets for Solution-Processible Electrodes and Conductive Coatings
}

Zach D. Seibers ${ }^{a^{*}}$, Elliot Brim ${ }^{a}$, Sandra Lee Pittelli ${ }^{a}$, Esther Beltran ${ }^{b}$, Meisha L. Shofner $^{\mathrm{c}}$, John R. Reynolds ${ }^{a}$

a. School of Chemistry and Biochemistry, School of Materials Science and Engineering, Center for Organic Photonics and Electronics (COPE), Georgia Tech Polymer Network (GTPN), Georgia Institute of Technology, Atlanta, Georgia 30332, United States.

b. Florida Space Institute, University of Central Florida, Office of Research and Commercialization, Orlando, FL, USA

c. School of Materials Science and Engineering, Georgia Institute of Technology, Atlanta, Georgia, 30332, United States.

*Corresponding author email: zachseibers@gmail.com

Table S1: Galactic Cosmic Ray Irradiation Schedule

\begin{tabular}{c|cc|cc}
\multirow{2}{*}{ Ion Species } & \multicolumn{2}{|c|}{ Acute Dose } & \multicolumn{2}{c}{ Low Dose } \\
\cline { 2 - 5 } & $\begin{array}{c}\text { Dose Rate } \\
\text { cGy/min }\end{array}$ & $\begin{array}{c}\text { Dose } \\
\text { cGy }\end{array}$ & $\begin{array}{c}\text { Dose Rate } \\
\text { cGy/min }\end{array}$ & $\begin{array}{c}\text { Dose } \\
\text { cGy }\end{array}$ \\
\hline P1000 & 18.5 & 28.00 & 0.41 & 28 \\
Si600 & 1.3 & 8.00 & 0.35 & 0.8 \\
He250 & 1.6 & 14.40 & 0.38 & 14.4 \\
O350 & 9.7 & 4.80 & 0.27 & 4.8 \\
Fe600 & 7.4 & .80 & 1.21 & 0.82 \\
P250 & 25.3 & 31.20 & 0.41 & 31.2 \\
\hline
\end{tabular}


Supporting Information

Table S2: Solar Particle Event Irradiation Schedule

\begin{tabular}{ccc} 
Proton Energies & \multicolumn{2}{c}{ Acute Dose } \\
\cline { 2 - 3 } MeV & $\begin{array}{c}\text { Dose Rate } \\
\text { CGy/min }\end{array}$ & $\begin{array}{c}\text { Dose } \\
\text { cGy }\end{array}$ \\
\hline $\mathbf{5 0}$ & 0.44 & 36.7 \\
$\mathbf{6 0}$ & 0.46 & 1.17 \\
\hline $\mathbf{7 0}$ & 0.44 & 0.81 \\
$\mathbf{8 0}$ & 0.39 & 0.60 \\
$\mathbf{9 0}$ & 0.42 & 0.42 \\
\hline $\mathbf{1 0 0}$ & 0.36 & 0.32 \\
\hline $\mathbf{1 1 0}$ & 0.33 & 0.22 \\
\hline $\mathbf{1 2 0}$ & 0.34 & 0.15 \\
\hline $\mathbf{1 3 0}$ & 0.34 & 0.11 \\
\hline $\mathbf{1 4 0}$ & 0.36 & 0.08 \\
\hline $\mathbf{1 5 0}$ & 0.26 & 0.06 \\
\hline
\end{tabular}




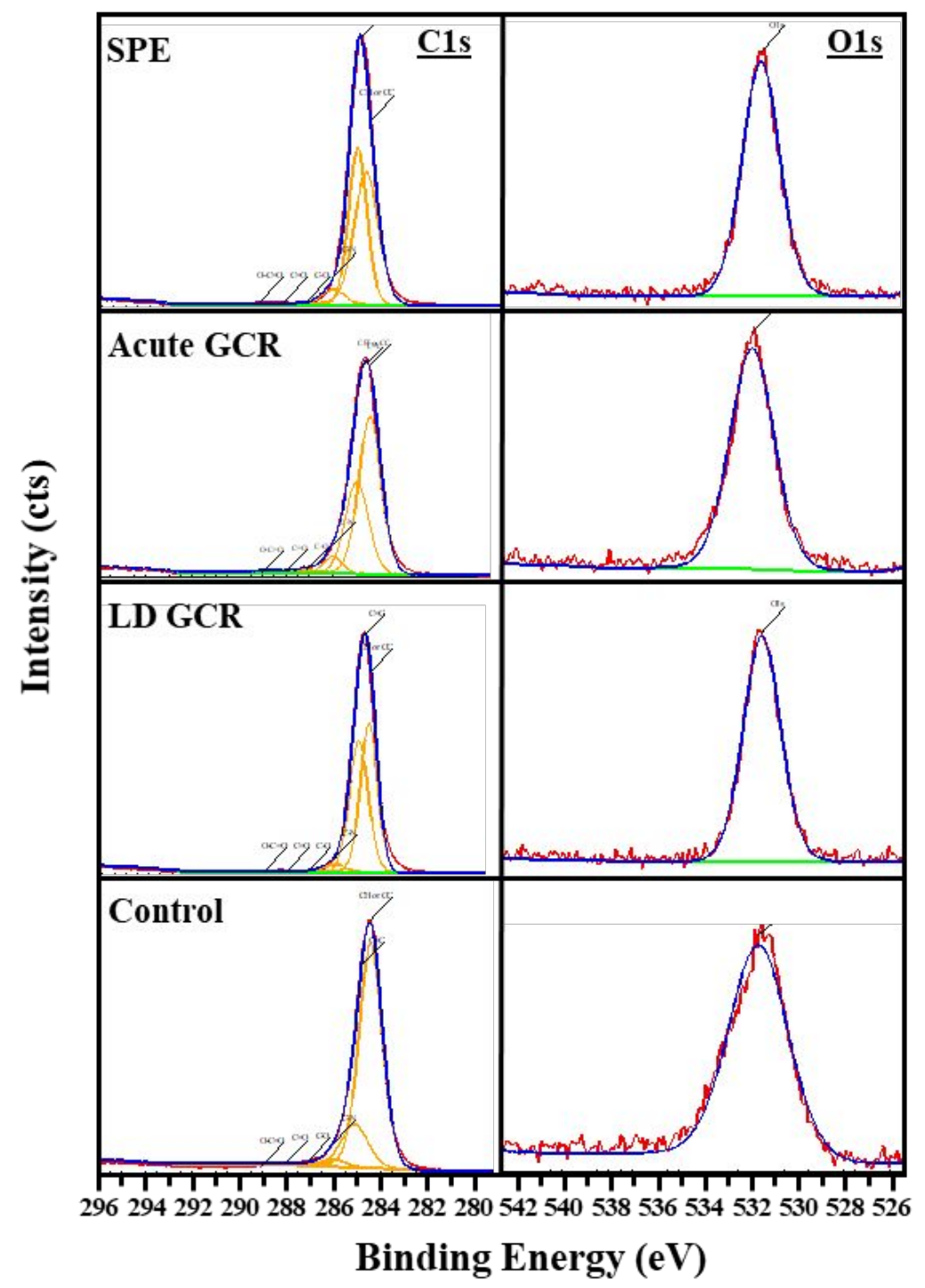

Figure S1: Spectra for XPS of irradiated rGO-dd laminate films atop HPDE. Columns correspond to peaks for $\mathrm{C} 1 \mathrm{~s}$ and $\mathrm{O} 1 \mathrm{~s}$ signals. Each row represents a different irradiation treatment including SPE, acute GCR, LD GCR, and a control. Each spectrum is dominated by large C peaks from the HDPE substrate that limit the sensitivity of the technique. No chemical changes are observed between the control and any of the irradiated samples. 